\title{
Methods used in mobile applications for the diagnosis of hearing loss: A systematic mapping study
}

\section{Métodos utilizados en aplicaciones móviles para al diagnóstico de pérdida auditiva: Un mapeo sistemático de literatura}

\section{Atencio Vizcaíno Hebert Leonidas, Tintín Perdomo Verónica Paulina, Caiza Caizabuano José Rubén, and Caicedo Altamirano Fernando Sebastián}

Universidad de las Fuerzas Armadas ESPE,Quijano Ordoñez y Hermanas Páez, Latacunga Ecuador

\section{Abstract}

Hearing loss is one of the most common health problems today, it can appear at any age and the causes are varied, in order to prevent it or adapt to the changes brought about by the hearing impairment, it is necessary to diagnose it in time. The technology in terms of applications for health care smartphones has constantly evolved, so that today play an important role and are among the most downloaded from application stores, several of these applications are the diagnosis of hearing loss and use the method of pure tones. In this study a Systematic Mapping of Literature SMS (Systematic Mapping Study) is made to look for mobile applications that use other diagnostic methods that offer similar or better results, of the 13 applications found, 11 used the method of pure tones and in only 2 of them was implemented the speech audiometry (word recognition), concludes that diagnostic hearing loss tests based on mobile applications are reliable alternatives to conventional audiometric systems, and that pure tone thresholds alone are an incomplete assessment of hearing, and there is a need to develop new hearing measurement methods and combine them with other methods to complement the diagnosis.

Resumen: La pérdida de la audición es uno de los problemas de salud más comunes en la actualidad, puede aparecer a cualquier edad y las causas son variadas, para poder prevenirla o adaptarse a los cambios que conlleva la deficiencia auditiva, es necesario diagnosticarla a tiempo. La tecnología en cuanto a aplicaciones para smartphones de asistencia de salud ha evolucionado constantemente, tal es así que hoy en día juegan un papel importante y son de las más descargadas de las tiendas de aplicaciones, varias de esas aplicaciones son las de diagnóstico de pérdida auditiva y utilizan el método de los tonos puros. En este estudio se hace un Mapeo Sistemático de Literatura SMS (Systematic Mapping Study) para buscar aplicaciones móviles que utilicen otros métodos de diagnóstico que ofrezcan similares o mejores resultados, de las 13 aplicaciones encontradas, 11 utilizaron el método de los tonos puros y en solo 2 de ellas se implementó la logoaudiometria (reconocimiento de palabras), por lo que se 
concluye que las pruebas de diagnóstico de pérdida auditiva basadas en aplicaciones móviles, son alternativas confiables a los sistemas de audiometría convencionales, y que los umbrales de tonos puros por sí solos son una evaluación incompleta de la audición, y existe la necesidad de desarrollar nuevos métodos de medición de audición y combinarlos con otros métodos para complementar el diagnóstico.

Keywords: app, smartphone, audiometry, diagnosis, hearing loss, hearing disabilities Palabras Clave: aplicación, smartphone, audiometría, diagnóstico, pérdida de audición, discapacidad auditiva

\section{Introducción}

La Organización Mundial de la Salud considera a la pérdida de audición como una de las discapacidades crónicas más frecuentes en todo el mundo, más del $5 \%$ de la población mundial, esto es 466 millones de personas, padece pérdida de audición discapacitante, de los cuales: 432 millones son adultos y 34 millones son niños. Se estima que, en 2050, más de 900 millones de personas, es decir, una de cada 10 sufrirá una pérdida de audición discapacitante [1].

A medida que se envejece, las funciones del cuerpo y los órganos sensoriales se deterioran. La capacidad auditiva empieza a deteriorarse a partir de los 20 años de manera gradual, misma que se hace más evidente a partir de los 50 años [2]. En la pérdida auditiva leve, la adaptación es muy eficaz en una fase temprana, por lo que es importante detectar los problemas auditivos en esta fase [2]--[4]. Por lo general, las personas no son conscientes del progreso continuo de deterioro auditivo y se acostumbran a vivir con dificultades auditivas, por lo que es necesario la utilización de audiómetros que ayuden a diagnosticar la pérdida auditiva en una fase temprana[2].. Los diagnósticos oportunos y precisos son fundamentales para prevenir las consecuencias de la pérdida de audición [5]. Para la realización de una audiometría convencional se requiere de equipos y entornos de prueba insonorizados de difícil acceso [6], del mismo modo, las alternativas a la audiometría convencional como por ejemplo: audiómetros automatizados y portátiles, establecen el estándar adecuado en el diagnóstico de la pérdida auditiva, y aunque estas alternativas son precisas, son costosas y requieren personal capacitado, adicionalmente, el acceso a este tipo de tecnología está limitado por aspectos financieros, geográficos y logísticos [5]-[8]. La audiometría automatizada 
es un método valioso para evaluar la pérdida auditiva en entornos con acceso limitado al personal de audiología [9], sin embargo, la necesidad de una audiometría más accesible y la ubicuidad de los dispositivos portátiles han fomentado el desarrollo de una variedad de aplicaciones móviles para la evaluación de la pérdida auditiva [5], [7], [10], [11]. Aunque estas nuevas aplicaciones son ampliamente accesibles y pueden ser utilizados por individuos sin formación formal, pocos han sido validados científicamente.[5], [7].

Las pruebas de audiometría automatizadas pueden ampliar el acceso a la audiometría diagnóstica, un smartphone con audífonos puede ser utilizado como un audiómetro accesible, de bajo costo y portátil que no requiere de un profesional capacitado para su uso [7], [12]. Las aplicaciones de pruebas auditivas son relativamente nuevas en el panorama de la salud móvil y utilizan hardware de teléfono interno estándar y un auricular de uso diario para evaluar la pérdida auditiva[13], [14].

Ahora bien, los diagnósticos oportunos y precisos son fundamentales para prevenir las consecuencias de la pérdida de audición [5]. Sin embargo, para realizar una audiometría convencional se requiere de equipos y entornos de prueba que generalmente son de difícil acceso [6]. Del mismo modo, las alternativas a la audiometría convencional como, por ejemplo: audiómetros automatizados y portátiles, solo permiten un diagnóstico estándar para determinar una pérdida auditiva. Aunque estas alternativas son precisas, también son costosas y requieren personal capacitado. Esto se agrava más cuando las personas con problemas auditivos no tienen acceso a este tipo de tecnología debido aspectos económicos, geográficos y logísticos [5], [8].

Estos aspectos nos permiten indagar acerca de las aplicaciones móviles para el diagnóstico de la pérdida auditiva y se plantea un mapeo sistemático de literatura para determinar cuántas aplicaciones móviles para pérdida auditiva hay, métodos de diagnóstico más comúnmente utilizados y características principales, para lo cual se consideraron estudios publicados a partir del año 2003 hasta el 2018.

El resto de este documento está estructurado de la siguiente manera. La Sección 2 presenta los pasos que se llevan a cabo en esta revisión sistemática, presentando las preguntas de investigación, el protocolo de investigación, la conducción y el proceso de extracción de datos. La sección 3 se presentan el resumen y análisis de los datos observados en los estudios seleccionados, respondiendo a las preguntas de investigación. La sección 4 presenta las amenazas a la validez relacionada con esta revisión sistemática y cómo minimizar el impacto. Finalmente, la Sección 5 se presenta la discusión de resultados, así como analizar aspectos relevantes encontrados de este estudio de investigación. 


\section{Antecedentes}

Uno de los regalos más preciados que la naturaleza les ha concedido a las personas es la comunicación. Por lo tanto, el lenguaje es la capacidad que adquiere el hombre, de poder expresar, intercambiar ideas y transmitir sus pensamientos, lo que al ser captadas a través de los sentidos codifican y decodifican la información que son almacenadas y controladas por el cerebro.

\section{1. ¿Qué es la discapacidad auditiva?}

Hoy en día se conoce por discapacidad auditiva lo que generalmente se ha considerado como sordera, también es utilizada para referirse a todos los tipos y grados de pérdida auditiva y por lo general es usada como sinónimo de deficiencia auditiva o hipoacusia y es un término amplio que comprende toda una serie de incapacidades que van desde lo superficial hasta lo más profundo [1]. Una minuciosa historia y examen clínico (incluyendo el uso de diapasones) son esenciales, y contribuyen a tener una buena idea de la situación antes de hacer las mediciones a describir y estas se hacen utilizando instrumentos electrónicos.

\subsection{Métodos de diagnóstico más comunes}

La evaluación auditiva se realiza a través de una serie de exámenes que permiten determinar el grado de pérdida auditiva y la localización del problema, esta evaluación es hoy por hoy tan compleja e importante dentro del campo de la otología y ha dado origen a una disciplina científica llamada audiología. También es posible realizar un diagnóstico utilizando instrumentos electrónicos incluidos los smartphones [1]. Dentro de los métodos más usuales están: audiometría clásica de tonos puros, evaluaciones especiales, logoaudiometría (audiometría verbal), audiometría a campo libre, audiometría de potenciales evocados auditivos, audiometría de emisiones otoacústicas e inmitancia acústica -- timpanometría, y dentro de los más comúnmente utilizados en audiología están:

Audiometría clásica de tonos puros:

Este es el examen fundamental para la medición de audición, se busca determinar el umbral (el estímulo de menor intensidad que es capaz de percibir el oído) para cada una de las frecuencias [15].

Logoaudiometría (audiometría verbal): 
En este método de diagnóstico se evalúa la perdida auditiva en base a la audición y comprensión del lenguaje mediante conducción aérea [15].

\section{Mapeo Sistemático de Literatura (Método)}

Un SMS (Systematic Mapping Study), es una metodología que consiste en investigar en la literatura, sobre un área de interés particular, con el objetivo de determinar la naturaleza el alcance y la cantidad de estudios primarios publicados, dando una visión general de un área de investigación a través de la clasificación y el conteo de las contribuciones [16].

En este estudio se sigue las directrices establecidas en la guía para realización de mapeos sistemáticos de literatura en ingeniería de software de Petersen [16]. El objetivo de este SMS es analizar aplicaciones móviles para el diagnóstico de pérdida auditiva, tomando como período de referencia desde el año 2003 hasta el 2018, lapso de tiempo en el cual se concentra la mayor parte de estudios sobre el desarrollo de este tipo de aplicaciones, para lo cual se busca responder a las siguientes preguntas de investigación $\mathrm{RQ}$ (Request Question).

RQ1: ¿Cuantas aplicaciones móviles utilizadas para diagnóstico de pérdida auditiva han sido identificadas en los estudios?

RQ2: ¿Que métodos de diagnóstico de pérdida auditiva se han utilizado para la implementación de las aplicaciones móviles?

RQ3: ¿De los métodos de diagnóstico de pérdida auditiva identificados, cual o cuales son los más comúnmente utilizados por las aplicaciones móviles?

RQ4: ¿Qué características principales tienen estas aplicaciones móviles?

En la literatura se han encontrado varias revisiones sistemáticas que abordan temas relacionados al desarrollo de aplicaciones móviles para diagnostico de perdida auditiva, pero estos estudios se centran únicamente en aplicaciones que utilizan el método de audiometría clásica de tonos puros, por lo que para este estudio se plantean preguntas de investigación que servirán para identificar si en la literatura se describen aplicaciones móviles que utilicen otros métodos de diagnóstico.

\subsection{Estrategia de Búsqueda}

Para la selección de estudios primarios es necesario definir la cadena de búsqueda en base a un conjunto de palabras clave, en este estudio se utiliza la estrategia PICO 
sugerida por Kitchemham en la Guía para la realización de revisiones sistemáticas de literatura en ingeniería de software [17] y utilizada también por Petersen en la Guía para la realización de mapeos sistemáticos en ingeniería de software [16].

Esta estrategia define los criterios de población, intervención, comparación y resultados, lo que permite ajustar las palabras clave que servirán para estructurar la cadena de búsqueda en las diferentes fuentes bibliográficas y poder responder las preguntas de investigación planteadas.

\subsubsection{Población}

En el contexto de este estudio, la población corresponde al conjunto de aplicaciones móviles para el diagnóstico de pérdida auditiva, se determinaron las siguientes palabras clave: "application" OR "tool" OR "app" OR "software" AND "smartphone".

\subsubsection{Intervención}

Manejo o intervención de interés que para este estudio constituye los métodos de diagnóstico utilizados en las aplicaciones móviles, palabra clave: "diagnosis" OR "detection" OR "examination" OR "test*" OR "audiometry" OR "measure".

\subsubsection{Comparación}

No se hace ninguna comparación empírica, es decir no existe una intervención alternativa con la cual comparar, aunque no siempre se dispone de la misma, por lo que se omite este componente y la estrategia PICO se convierte en PIO.

\subsubsection{Resultados}

Es la consecuencia relevante de interés, el resultado esperado es el diagnóstico de la pérdida auditiva, palabras clave: "hearing loss" OR "hearing disabilities" OR "hearing level".

Para la búsqueda de los estudios primarios se utilizaron bases de datos bibliográficas de resúmenes y citas de artículos de revistas científicas con títulos de editores internacionales, incluyendo la cobertura de revistas revisadas por pares de las áreas de ciencias, tecnología, medicina, ciencias sociales, artes y humanidades como: Scopus, Web of Science y ScienceDirect: y Medline, una base de datos bibliográfica medica 
que incluye artículos científicos publicados en revistas médicas, a la cual es posible acceder a través de PubMed. Entonces, la cadena de búsqueda se construyó en base a la estructuración de las expresiones regulares, formada por las palabras claves determinadas en la estratega PICO y enlazadas con los conectores lógicos AND y OR aplicada a los títulos y abstracts. Dando como resultado la siguiente cadena de búsqueda, en la tabla 1 se puede observar los resultados obtenidos según la base digital.

(("application" OR "tool" OR "app" OR "software") AND ("mobile" OR OR "iphone" OR "smart-phone" OR "smart mobile") AND ("diagnosis" OR "detection" OR "examination" OR "test*" OR "audiometry" OR "measure") AND ("hearing loss" OR "hearing disabilities" OR "hearing level"))

TABLE 1: Número de Estudios Encontrados.

\begin{tabular}{l|c|}
\hline Fuente Bibliográfica & $\begin{array}{c}\text { Estudios } \\
\text { Encontrados }\end{array}$ \\
\hline Scopus & 75 \\
\hline Science Direct & 3 \\
\hline PubMed & 25 \\
\hline Web of Science & 62 \\
\hline Total & 165 \\
\hline
\end{tabular}

\subsection{Selección de Estudios}

La selección de estudios se realizó en base a las palabras claves, sinónimos y términos relacionados en el título y resúmenes, tomando en consideración estudios en los que el método de evaluación de diagnóstico de la pérdida auditiva sea el reconocimiento de palabras principalmente, para lo cual se han definido los siguientes criterios de inclusión (Cl) y de exclusión (CE).

Criterios de inclusión de artículos:

Cl1: Estudios en cuyo contenido se haga referencia a aplicaciones móviles para el diagnóstico de pérdida auditiva.

$\mathrm{Cl}$ 2: Estudios en los que se identifique el método de diagnóstico implementado en la aplicación móvil.

Cl3: Estudios en el que se identifique el tipo de transducción utilizado para el diagnóstico.

Cl4: Estudios que describan características adicionales de las aplicaciones móviles.

Criterios de exclusión de artículos: 
CE1: Estudios en los que no se identifique la aplicación móvil para diagnóstico.

CE2: Estudios en los que no especifiquen el método de diagnóstico utilizado.

CE3: Estudios que no muestren características relevantes de las aplicaciones.

CE4: Estudios que solamente sean casos de prueba de aplicaciones comerciales.

De los resultados de la búsqueda en las diferentes fuentes bibliográficas, se obtuvo un total de 165 estudios publicados entre el año 2003 hasta el año 2018, periodo de tiempo durante el cual se desarrollaron aplicaciones para el diagnóstico de pérdida auditiva de propósito general, en los cuales se han utilizado diferentes métodos de diagnóstico.

La búsqueda se ejecutó con la finalidad de obtener una lista de estudios preseleccionados, la gestión de la bibliografía se llevó a cabo mediante el uso de un gestor bibliográfico. El procedimiento para la preselección de los estudios fue aplicado a todos los artículos obtenidos con el fin de verificar si los estudios se ajustan a los criterios de inclusión y exclusión y se centró en encontrar las palabras clave en títulos, resúmenes. De los 165 estudios encontrados se descargaron 107 estudios, 58 artículos fueron descartados, de los cuales, 57 fueron duplicados y 1 estaba en otro idioma. El detalle se puede observar en la Tabla 2.

TABLE 2: Artículos Preseleccionados Según Fuente.

\begin{tabular}{l|c|c|c|c|}
\hline Base de Datos & Encontrados & Duplicados & Otro Idioma & Preseleccionados \\
\hline Scopus & 75 & 22 & & 53 \\
\hline Science Direct & 3 & 1 & 1 & 1 \\
\hline PubMed & 25 & 12 & & 40 \\
\hline Web of Science & 62 & 22 & & 107 \\
\hline Total & 165 & & & \\
\hline
\end{tabular}

\subsection{Extracción de Datos}

Para poder determinar la cantidad de estudios que cumplen con los criterios de inclusión y poder responder a las preguntas de investigación, del total de publicaciones extraídas de las bases digitales (107 preseleccionados), de cada publicación fue necesario extraer información relevante con la ayuda del gestor bibliográfico y se generó un reporte con los datos que se muestran en la Tabla 3: 
TABLE 3: Elementos de Datos Extraídos.

\begin{tabular}{l|l} 
Datos Extraídos & $\begin{array}{l}\text { Descripción } \\
\text { Type }\end{array}$ \\
$\begin{array}{l}\text { Tipo de articulo, articulo de conferencia, artículo de } \\
\text { revista, sección de libro, manuscrito } \\
\text { Author }\end{array}$ & Nombres de los autores del documento \\
\hline Date & Fecha de publicación del documento \\
\hline DOI & Identificador del objeto digital \\
\hline Lybrary Catalog & Base digital donde está alojado el artículo \\
\hline Conference Name & Nombre de la conferencia donde fue presentado \\
\hline Abstract & Resumen del artículo \\
\hline Short Title & Título corto \\
\hline Date Added & Fecha de adición del articulo a la base \\
Modified & Fecha de última modificación \\
\hline Tags & Palabras clave
\end{tabular}

\subsection{Análisis, Clasificación y Selección}

Para la selección de artículos primarios se realizó la lectura de los resúmenes de los artículos preseleccionados en la fase anterior, se seleccionaron aquellos que, según su contenido, vayan a dar respuesta a las preguntas de investigación planteadas, y en base al análisis y clasificación se seleccionaron de las bases según lo detallado en la Tabla 4.

TABLE 4: Artículos Seleccionados.

Fuente Bibliográfica
Scopus
Science Direct
PubMed
Web of Science
Total

\begin{tabular}{|c|}
\hline Preseleccionados \\
\hline 75 \\
\hline 3 \\
25 \\
62 \\
165 \\
\hline
\end{tabular}

\begin{tabular}{|c|}
\hline Seleccionados \\
\hline 26 \\
1 \\
2 \\
0 \\
29 \\
\hline
\end{tabular}

Para la selección de los 29 estudios primarios, en este estudio se realiza una modificación de la declaración PRISMA (Preferred Reporting Items for Systematic Reviews and Meta-Analyses), que establece la práctica adecuada para informar resultados de una búsqueda de revisiones sistemáticas [18]. En la Figura 1 se proporciona una descripción del proceso que se siguió para seleccionar los estudios utilizando los criterios de elegibilidad especificados previamente. 


\begin{tabular}{|c|c|c|c|c|}
\hline \multirow{4}{*}{ 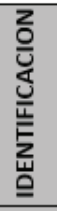 } & \multicolumn{4}{|c|}{ NUMERO DE REGISTROS IDENTIFICADOS EN LAS BASES } \\
\hline & SCOPUS & SCIENCE DIRECT & MEDLINE & WEB OF SCIENCE \\
\hline & $\mathrm{n}=75$ & $\mathrm{n}=3$ & $n=25$ & $n=62$ \\
\hline & \multicolumn{4}{|c|}{$\mathrm{n}=165$} \\
\hline \multirow{8}{*}{ 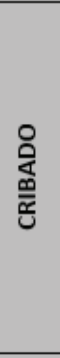 } & \multicolumn{4}{|c|}{ NUMERO DE REGISTROS TRAS ELIMINAR DUPLICADOS } \\
\hline & SCOPUS & SCIENCE DIRECT & MEDLINE & WEB OF SCIENCE \\
\hline & $n=53$ & $\mathrm{n}=2$ & $\mathrm{n}=13$ & $n=40$ \\
\hline & \multicolumn{4}{|c|}{$\mathrm{n}=108$} \\
\hline & \multicolumn{4}{|c|}{ NUMERO DE RESGISTROS TRAS ELIMINAR ARTICULOS EN OTRO IDIOMA } \\
\hline & IEEE & SCOPUS & SPRINGER & WEB OF SCIENCE \\
\hline & $\mathrm{n}=0$ & $\mathrm{n}=0$ & $\mathrm{n}=1$ & $\mathrm{n}=0$ \\
\hline & & & & \\
\hline \multirow{2}{*}{ 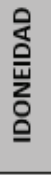 } & \multicolumn{2}{|c|}{$\begin{array}{l}\text { ARTICULOS DE TEXTO COMPLETO } \\
\text { EVALUADOS PARA SU ELEGIBILIDAD }\end{array}$} & \multicolumn{2}{|c|}{$\begin{array}{l}\text { ARTICULOS DE TEXTO COMPLETO } \\
\text { EXCLUIDOS CON SUS RAZONES }\end{array}$} \\
\hline & \multicolumn{2}{|r|}{$n=107$} & \multicolumn{2}{|c|}{ No responden a las $R Q \mathrm{n}=78$} \\
\hline \multirow{2}{*}{$\begin{array}{l}z \\
\text { o } \\
\stackrel{3}{J} \\
\underline{u} \\
\underline{z}\end{array}$} & \multicolumn{4}{|c|}{$\begin{array}{l}\text { NUMERO DE ESTUDIOS INCLUIDOS EN LA SINTESIS CUALITATIVA Y } \\
\text { CUANTITATIVA }\end{array}$} \\
\hline & \multicolumn{4}{|c|}{$n=29$} \\
\hline
\end{tabular}

Figure 1: Modelo PRISMA para la selección de estudios primarios.

\subsection{Verificación de la Calidad de los Estudios}

Para la verificación de la calidad de los estudios se verificó si se cumple con los siguientes criterios:

VC1: ¿El estudio seleccionado contribuye a dar respuestas a las preguntas de investigación?

VC2: ¿El estudio seleccionado contiene referencias a estudios publicados en revistas, conferencias o congresos?

VC3: ¿El estudio está basado en una investigación?

VC4: ¿Existe una declaración clara de los objetivos de la investigación?

VC5: ¿Hay una declaración clara de los resultados?

VC6: ¿El estudio seleccionado está escrito en el idioma inglés?

\section{Resultados}

En la Tabla 5 se puede visualizar los artículos seleccionados según el tipo de estudio, del total de artículos seleccionados, el 90\% $(n=26)$ corresponden a artículos de revistas 
científicas, el 7\% ( $n=2)$ corresponden a artículos de conferencia y el 3\% ( $n=1)$ corresponde a un manuscrito.

TABLE 5: Distribución de Artículos Según el Tipo de Articulo.

$\begin{array}{ll}\text { Artículo } & \text { Estudios Seleccionados } \\ \begin{array}{l}\text { De revista } \\ \text { científica }\end{array} & {[19],[20],[9],[21],[22],[23],[24],[25],[6],[14],[26],[27],} \\ & {[11],[28],[29],[4],[30],[5],[31],[32],[12],[13],[33],[34],} \\ \text { De conferencia } & {[35],[36]}\end{array}$
$\begin{aligned} & \text { Manuscrito } \\ & \text { Man] }[38]\end{aligned}$

El protocolo de investigación más utilizado es el estudio de caso, el 69\% ( $n=20)$ de los estudios primarios que utilizan este protocolo hacen referencia a aplicaciones móviles de diagnóstico de pérdida auditiva ya existentes en el mercado; en ciencia del diseño, el $28 \%(n=8)$ de los estudios primarios corresponden a desarrollo en implementación de aplicaciones móviles nuevas que no están disponibles en el mercado de aplicaciones; y el 3\% ( $n=1)$ es una revisión sistema de literatura de aplicaciones móviles para diagnóstico de pérdida auditiva disponibles en las plataformas de distribución digital en línea como Google Play Store y Apple App Store, donde el método de diagnóstico más utilizado es el tonos puros, el detalle se puede observar en la Tabla 6.

TABLE 6: Artículos Según Protocolo de Investigación.

\begin{tabular}{ll}
$\begin{array}{l}\text { Contribución } \\
\begin{array}{l}\text { Ciencia del } \\
\text { diseño }\end{array}\end{array}$ & Estudios Seleccionados \\
\hline $\begin{array}{l}\text { Estudio de caso } \\
\text { [16], [8], [18], [19], [22], [5], [13], [23], [10], [3], [27], [4], [28], } \\
\text { [29], [12], [30], [31], [32], [36], [25] }\end{array}$ \\
$\begin{array}{l}\text { Revisión de } \\
\text { literatura }\end{array}$ & {$[33]$}
\end{tabular}

Tomando como referencia el período comprendido entre los años 2003 y 2018, tiempo durante en el cual se desarrollaron y evaluaron aplicaciones móviles para diagnóstico de pérdida auditiva, se encontró que del total de estudios seleccionados: el $21 \%$ se publicaron en el 2016 , el $24 \%$ se publicaron en el 2017 y el $17 \%$ se publicaron hasta mayo del 2018, años en los que se presentaron un mayor número de publicaciones, con estudios de caso de aplicaciones móviles disponibles en el mercado online y, publicaciones donde se desarrollaron nuevas aplicaciones para el diagnóstico de pérdida auditiva, que no han sido comercializadas, en la Figura 2 se puede observar la distribución de artículos por año de publicación.

Para la identificación de aplicaciones se consideraron estudios en los cuales se describan aplicaciones móviles desarrolladas para el diagnóstico de pérdida auditiva y que estén disponibles en las tiendas de aplicaciones, donde se especifique el método 


\section{Artículos por año}

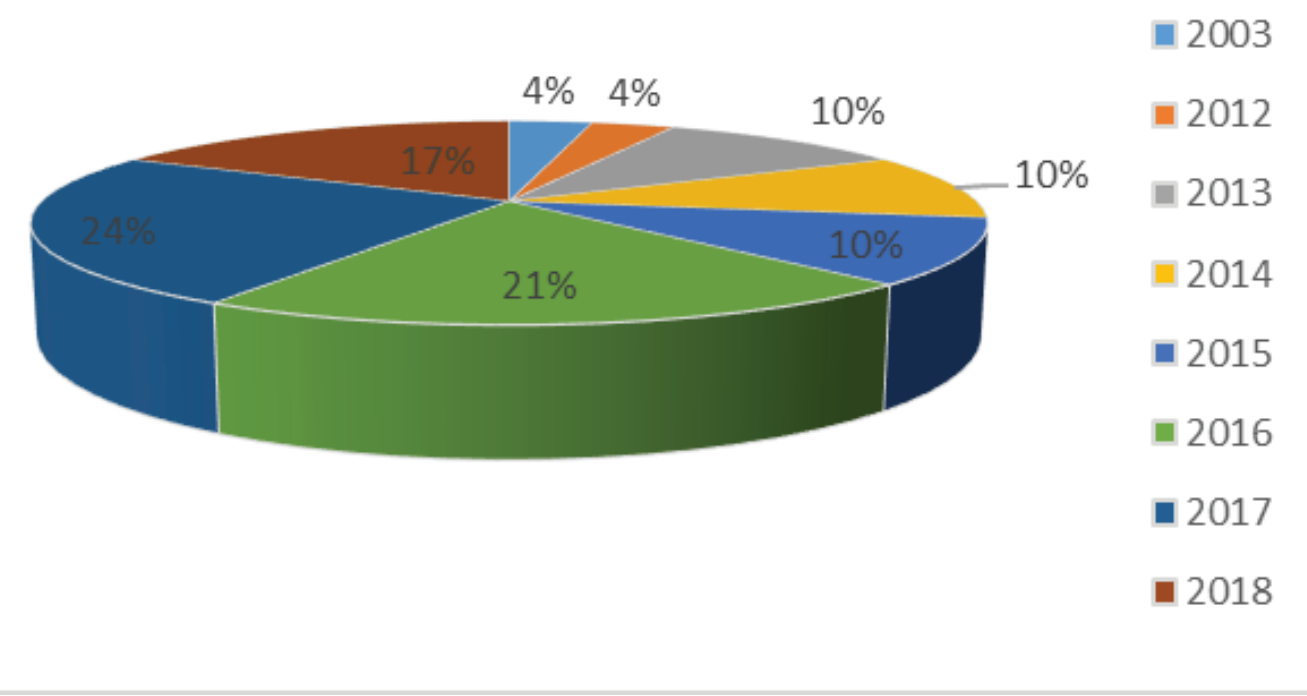

Figure 2: Artículos por año de publicación.

de diagnóstico y el tipo de transducción; se excluyó 1 estudio por corresponder a una revisión sistemática de literatura donde se analizó aplicaciones móviles disponibles en tiendas online únicamente, quedando 28 estudios en los que se utilizan este tipo de aplicaciones móviles, de las cuales se las identifica por su nombre comercial, 3 de ellas aun no tienen denominación y no están disponibles comercialmente.

TABLE 7: Aplicaciones Encontradas.

Aplicaciones móviles
Agilis Health Mobile Audiogram
AudCal
EarTrumpet
Hearing Test TM
hearScreen TM
MobileAudiometer
PhoSHA
S/N
UHear
VIA
WordRec
Total general

\begin{tabular}{|c|}
\hline Estudios que las incluyen \\
\hline 1 \\
\hline 2 \\
\hline 3 \\
\hline 3 \\
\hline 7 \\
\hline 1 \\
\hline 1 \\
\hline 3 \\
\hline 5 \\
\hline 1 \\
\hline 1 \\
\hline 28 \\
\hline
\end{tabular}




\section{Discusión}

Las pruebas de diagnóstico de pérdida auditiva basadas en aplicaciones móviles, son alternativas confiables a los sistemas de audiometría convencionales [9], [7]. Aunque útiles, los umbrales de tonos puros por sí solos son una evaluación incompleta de la audición, y existe la necesidad de desarrollar nuevos métodos de medición de audición y combinarlos con otros como la logoaudiometría para complementar el diagnóstico.

RQ1: ¿Cuantas aplicaciones móviles utilizadas para diagnóstico de pérdida auditiva han sido identificadas en los estudios?

En los 28 estudios primarios seleccionados se identificaron 13 aplicaciones móviles de diagnóstico de pérdida auditiva comerciales y desarrolladas, diseñadas para las plataformas Android e iOS, de las cuales, 5 de ellas se pueden descargar de Google Play Store y Apple App Store respectivamente, todas estas aplicaciones se encuentran en estudios que han sido validadas por pares, esto demuestra que en el campo del desarrollo de las aplicaciones móviles, el diagnóstico de pérdida auditiva es ampliamente utilizado.

RQ2: ¿Que métodos de diagnóstico de pérdida auditiva se han utilizado para la implementación de las aplicaciones móviles?

La evaluación auditiva se realiza a través de una serie de métodos que permiten determinar el grado de perdida de la audición, en las aplicaciones móviles encontradas en los estudios primarios se utilizan la audiometría clásica de tonos puros y la logoaudiometría, de las 13 aplicaciones identificadas, el 69\% de ellas utilizan el método de audiometría de tonos puros, el $23 \%$ de ellas utilizan la logoaudiometría y el $8 \%$ utilizan los dos métodos.

RQ3: ¿De los métodos de diagnóstico de pérdida auditiva identificados, cual o cuales son los más comúnmente utilizados por las aplicaciones móviles?

El método más utilizado por apps comerciales y no comerciales es la audiometría de tonos puros donde se mide el umbral de audición de conducción aérea y de conducción ósea en base a varias frecuencias, solo en 3 aplicaciones se utiliza la logoaudiometría donde se evalúa la audición y la comprensión del lenguaje y se hace por conducción aérea. Una de estas aplicaciones móviles como es AudCal utiliza los dos métodos de con lo que se aumenta la efectividad del diagnóstico.

Los tonos puros generados electrónicamente por un audiómetro, se envían a través de fonos (conducción aérea) o de vibradores óseos (conducción ósea), 8 de las 13 aplicaciones encontradas utilizan transducción por conducción aérea, 2 de las aplicaciones utilizan transducción por conducción aérea y transducción por conducción 


\section{Metodos de audiometría utilizados en} apps

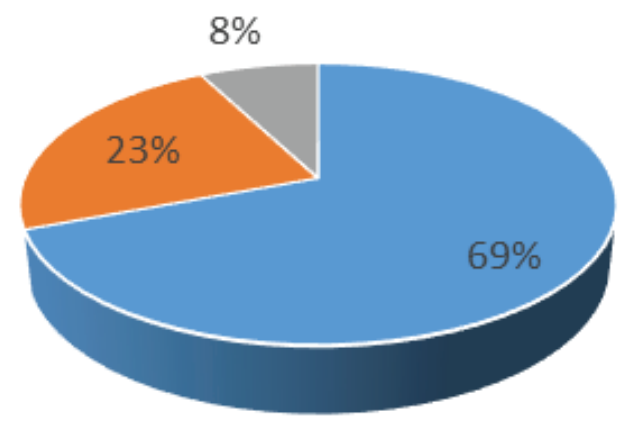

- Audiometría de tonos puros

- Logoaudiometría

- Los dos métodos

Figure 3: Métodos de diagnóstico de pérdida auditiva.

ósea simultáneamente y en 3 de ellas no se especifica el tipo de transducción. Con la utilización del método de tonos puros se mide en decibeles, el umbral de audición en todas las frecuencias, la audiometría de tonos puros también conocida como PTA, necesita un tiempo de prueba relativamente largo porque los sujetos necesitan responder repetidamente a muchos estímulos en diferentes frecuencias y niveles de sonido, de tal forma que es necesario utilizar otros métodos de diagnóstico como la logoaudiometría o audiometría verbal donde se evalúa el umbral de recepción de lenguaje y el reconocimiento de palabras.

RQ4: ¿Qué características principales tienen estas aplicaciones móviles?

De las 13 aplicaciones encontradas en los estudios seleccionados, cinco estudios fueron validadas por pares en cuanto a la efectividad y la asertividad del diagnóstico y pueden ser descargadas de las tiendas online, estas aplicaciones son: AudCal, EarTrumpet, Hearing Test, hearScreen y uHear; 8 aplicaciones fueron desarrolladas específicamente para pruebas de diagnóstico en cada estudio encontrado y aún no han sido comercializadas, estas son: Agilis Health Mobile Audiogram, MobileAudiometer, PhoSHA, VIA, WordRec y 3 de estas apps aún no tienen denominación [12], [20], [23], [24], [27], [29], [37], [38].

La aplicación que más se utilizó en estudios de casos fueron hearScreen [14], [22], [28], [30], [31], [32], [33], por ser una opción de audiometría accesible y de bajo costo en relación a la audiometría convencional, demostrando con esto que las aplicaciones móviles son una alternativa confiable para el diagnóstico de pérdida auditiva en una etapa inicial. De las mencionadas hay que destacar que dos de estas aplicaciones 
utilizan el método de reconocimiento de palabras, en especial PhoSHA que incorpora en su método de diagnóstico los fonemas coreanos.

De las aplicaciones encontradas, PhoSHA es adecuado para la estimación de audiogramas con suficiente precisión y tiempo de prueba reducido [20], y se basa principalmente en el diagnóstico de umbral de recepción de lenguaje con reconocimiento fonemas coreanos, WordRec utiliza el método del reconocimiento de palabras donde es necesario que la persona reconozca al menos el 50\% de una lista de palabras fonéticamente balanceadas y que representen lo mejor posible el espectro del lenguaje [12], en un último estudio se desarrolla una aplicación móvil que aún no tiene denominación y que utiliza el método o de reconocimiento verbal en base al reconocimiento de 120 dígitos triples en un ambiente con ruido[27].

TABLE 8: Características de las Aplicaciones Encontradas.

\begin{tabular}{|c|c|c|c|c|}
\hline Aplicación & Sistema Operativo & Comercial & $\begin{array}{l}\text { Método De } \\
\text { Evaluación }\end{array}$ & $\begin{array}{c}\text { Tipo De } \\
\text { Transducción }\end{array}$ \\
\hline $\begin{array}{l}\text { Agilis Health } \\
\text { Mobile Audiogram }\end{array}$ & Apple iOS & No & Tonos puros & $\begin{array}{l}\text { Conducción } \\
\text { Aérea }\end{array}$ \\
\hline AudCal & $\begin{array}{l}\text { Apple iOS } \\
\text { Android }\end{array}$ & $\mathrm{Si}$ & Tonos puros SRT & $\begin{array}{l}\text { Conducción } \\
\text { Aérea } \\
\text { Conducción Ósea }\end{array}$ \\
\hline EarTrumpet & Apple iOS & Si & Tonos puros & $\begin{array}{l}\text { Conducción } \\
\text { Aérea }\end{array}$ \\
\hline Hearing Test TM & Android & $\mathrm{Si}$ & Tonos puros & No especifica \\
\hline HearScreen TM & Android & $\mathrm{Si}$ & Tonos puros & $\begin{array}{l}\text { Conducción } \\
\text { Aérea }\end{array}$ \\
\hline $\begin{array}{l}\text { Mobile } \\
\text { Audiometer }\end{array}$ & No especifica & No & Tonos puros & $\begin{array}{c}\text { Conducción } \\
\text { Aérea }\end{array}$ \\
\hline PhoSHA & Android & No & $\begin{array}{l}\text { Fonemas } \\
\text { coreanos }\end{array}$ & $\begin{array}{l}\text { Conducción } \\
\text { Aérea }\end{array}$ \\
\hline $\mathrm{S} / \mathrm{N} 1$ & Multiplataforma & No & Tonos puros & No especifica \\
\hline $\mathrm{S} / \mathrm{N} 2$ & Android & No & Tonos puros & No especifica \\
\hline $\mathrm{S} / \mathrm{N} 3$ & Android & No & SRT & $\begin{array}{c}\text { Conducción } \\
\text { Aérea }\end{array}$ \\
\hline UHear & Apple iOS & $\mathrm{Si}$ & Tonos puros & $\begin{array}{l}\text { Conducción } \\
\text { Aérea }\end{array}$ \\
\hline VIA & Android & No & No especifica & $\begin{array}{l}\text { Conducción } \\
\text { Aérea } \\
\text { Conducción Ósea }\end{array}$ \\
\hline WordRec & Apple iOS & No & $\begin{array}{c}\text { WRS } \\
\text { Reconocimiento } \\
\text { de palabras }\end{array}$ & $\begin{array}{l}\text { Conducción } \\
\text { Aérea }\end{array}$ \\
\hline
\end{tabular}

El estudio demuestra que hay herramientas móviles efectivas para medir la pérdida de audición que se pueden descargar fácilmente de tiendas online y otras que se han 
desarrollado con las que se pueden realizar estudios confiables y de bajo costo ya que no difieren significativamente de las audiometrías convencionales.

El diagnóstico de pérdida auditiva utilizando smartphones es una opción ampliamente disponible y de bajo costo frente a la audiometría convencional. En estos estudios se demuestran que hay varias aplicaciones disponibles en el mercado online y además de otras que se desarrollan con este propósito, que son útiles para el diagnóstico en fases tempranas. Sin embargo, se requiere que estas aplicaciones amplíen su funcionalidad utilizando al menos dos métodos de diagnóstico, como los mencionados en este estudio con el propósito de ampliar la precisión de las evaluaciones de audiometría. A pesar de las ventajas de este tipo de aplicaciones como: la portabilidad, la accesibilidad, la autoadministración y el bajo, estas no remplazan a las pruebas de audiometría convencional realizadas por un audiólogo, pero ofrecen una opción funcional para aquellas personas con poco acceso a la audiometría convencional considerándose una opción confiable ara detección temprana de pérdida auditiva.

\section{Agradecimientos}

A la Universidad de las Fuerzas Armadas ESPE, por la organización del taller de Elaboración de Revisiones Sistemáticas y a sus instructores por el tiempo y el esfuerzo dedicado para la realización del presente trabajo.

\section{References}

[1] «Sordera y pérdida de la audición». [En línea]. Disponible en: https://www.who.int/es/ news-room/fact-sheets/detail/deafness-and-hearing-loss. [Accedido: 29-ago-2019].

[2] N. Nakamura, «Development of "MobileAudiometer" for screening using mobile phones», presentado en Annual International Conference of the IEEE Engineering in Medicine and Biology - Proceedings, 2004, vol. 26 V, pp. 3369-3372.

[3] H. Yousuf, S. Wet, de J. Biagio, H. C. Myburgh, R. H. Eikelboom, y J. Hugo, «Smartphone hearing screening in mHealth assisted community-based primary care», Journal of Telemedicine and Telecare, vol. 22, n. ${ }^{\circ}$ 7, pp. 405-412, 2016.

[4] L. Livshitz et al., «Application-Based Hearing Screening in the Elderly Population», Annals of Otology, Rhinology and Laryngology, vol. 126, n. ${ }^{\circ}$ 1, pp. 36-41, 2017.

[5] J. Saliba, M. Al-Reefi, J. S. Carriere, N. Verma, C. Provencal, y J. M. Rappaport, «Accuracy of Mobile-Based Audiometry in the Evaluation of Hearing Loss in Quiet 
and Noisy Environments», Otolaryngology - Head and Neck Surgery (United States), vol. 156, n. ${ }^{\circ}$ 4, pp. 706-711, 2017.

[6] S. Derin, O. H. Cam, H. Beydilli, E. Acar, S. S. Elicora, y M. Sahan, «Initial assessment of hearing loss using a mobile application for audiological evaluation», Journal of Laryngology and Otology, vol. 130, n. ${ }^{\circ}$ 3, pp. 248-251, 2016.

[7] S. Abu-Ghanem, O. Handzel, L. Ness, M. Ben-Artzi-Blima, K. Fait-Ghelbendorf, y M. Himmelfarb, «Smartphone-based audiometric test for screening hearing loss in the elderly», European Archives of Oto-Rhino-Laryngology, vol. 273, n. ${ }^{\circ}$ 2, pp. 333-339, 2016.

[8] Y. Liu, D. Yang, F. Xiong, L. Yu, F. Ji, y Q.-J. Wang, «Development and validation of a portable hearing self-testing system based on a notebook personal computer», Journal of the American Academy of Audiology, vol. 26, n. ${ }^{\circ}$ 8, pp. 716-723, 2015

[9] A. Foulad, P. Bui, y H. Djalilian, «Automated audiometry using Apple iOS-based application technology», Otolaryngology - Head and Neck Surgery (United States), vol. 149, n. ${ }^{\circ}$ 5, pp. 700-706, 2013.

[10] M. Coleman, «There's a hearing app for that», The Hearing Journal, vol. 64, n. ${ }^{o} 11, \mathrm{p}$. 12, nov. 2011.

[11] L. Renda, Ö. T. Selçuk, H. Eyigör, Ü. Osma, y M. D. Yılmaz, «Smartphone based audiometric test for confirming the level of hearing; is it useable in underserved areas?», Journal of International Advanced Otology, vol. 12, n. ${ }^{o}$ 1, pp. 61-66, 2016.

[12] N. A. Dewyer, P. Jiradejvong, S. Henderson, y C. J. Limb, «Automated smartphone audiometry: Validation of a word recognition test app», Laryngoscope, vol. 128, n. ${ }^{o}$ 3, pp. 707-712, 2018.

[13] R. K. V. Sethi, I. Ghanad, V. Kanumuri, B. Herrmann, E. D. Kozin, y A. K. Remenschneider, «Mobile Hearing Testing Applications and the Diagnosis of Sudden Sensorineural Hearing Loss: A Cautionary Tale», Otology and Neurotology, vol. 39, n. ${ }^{\circ}$ 1, pp. e1-e4, 2018.

[14] F. Mahomed-Asmail, D. W. Swanepoel, R. H. Eikelboom, H. C. Myburgh, y J. Hall III, «Clinical Validity of hearScreen ${ }^{T M}$ Smartphone Hearing Screening for School Children», Ear and Hearing, vol. 37, n. ${ }^{\circ}$ 1, pp. e11-e17, 2016.

[15] «MetodosEvaluacionAuditiva.pdf». [En línea]. Disponible en: http://www.clc. cl/clcprod/media/contenidos/pdf/MED_14_4/MetodosEvaluacionAuditiva.pdf. [Accedido: 29-ago-2019].

[16] K. Petersen, S. Vakkalanka, y L. Kuzniarz, «Guidelines for conducting systematic mapping studies in software engineering: An update», Information and Software Technology, vol. 64, pp. 1-18, ago. 2015. 
[17] B. Kitchenham, «Procedures for Performing Systematic Reviews», p. 33.

[18] A. Liberati et al., «The PRISMA Statement for Reporting Systematic Reviews and Meta-Analyses of Studies That Evaluate Health Care Interventions: Explanation and Elaboration», PLOS Medicine, vol. 6, n.o 7, p. e1000100, jul. 2009.

[19] T. Harris, S. Peer, y J. J. Fagan, «Audiological monitoring for ototoxic tuberculosis, human immunodeficiency virus and cancer therapies in a developing world setting», Journal of Laryngology and Otology, vol. 126, n. ${ }^{\circ}$ 6, pp. 548-551, 2012.

[20] J. M. Choi, J. Sohn, Y. Ku, D. Kim, y J. Lee, «Phoneme-based self hearing assessment on a smartphone», IEEE Journal of Biomedical and Health Informatics, vol. 17, n. ${ }^{o} 3$, pp. 526-529, 2013.

[21] O. Handzel, O. Ben-Ari, D. Damian, M. M. Priel, J. Cohen, y M. Himmelfarb, «Smartphone-based hearing test as an aid in the initial evaluation of unilateral sudden sensorineural hearing loss», Audiol. Neurootol., vol. 18, n. ${ }^{\circ}$ 4, pp. 201-207, 2013.

[22] D. W. Swanepoel, H. C. Myburgh, D. M. Howe, F. Mahomed, y R. H. Eikelboom, «Smartphone hearing screening with integrated quality control and data management», Int J Audiol, vol. 53, n. ${ }^{\circ}$ 12, pp. 841-849, dic. 2014.

[23] Y. Na, H. S. Joo, H. Yang, S. Kang, S. H. Hong, y J. Woo, «Smartphone-based hearing screening in noisy environments», Sensors (Basel), vol. 14, n. ${ }^{\circ}$ 6, pp. 10346-10360, jun. 2014

[24] F. Larrosa et al., «Development and evaluation of an audiology app for iPhone/iPad mobile devices», Acta Otolaryngol., vol. 135, n. ${ }^{\circ}$ 11, pp. 1119-1127, 2015.

[25] S. Peer y J. J. Fagan, «Hearing loss in the developing world: evaluating the iPhone mobile device as a screening tool», S. Afr. Med. J., vol. 105, n. ${ }^{\circ}$ 1, pp. 35-39, ene. 2015.

[26] M. Masalski, L. Kipinski, T. Grysinski, y T. Krecicki, «Hearing Tests on Mobile Devices: Evaluation of the Reference Sound Level by Means of Biological Calibration», J. Med. Internet Res., vol. 18, n. ${ }^{\circ}$ 5, p. e130, may 2016.

[27] J.-M. Potgieter, D. W. Swanepoel, H. C. Myburgh, T. C. Hopper, y C. Smits, «Development and validation of a smartphone-based digits-in-noise hearing test in South African English», Int J Audiol, vol. 55, n. ${ }^{\circ}$ 7, pp. 405-411, 2015.

[28] S. Y. Hussein, D. W. Swanepoel, L. B. de Jager, H. C. Myburgh, R. H. Eikelboom, y J. Hugo, «Smartphone hearing screening in mHealth assisted community-based primary care», J. Telemed. Telecare, vol. 22, n. ${ }^{0}$ 7, pp. 405-412, oct. 2016.

[29] A. Kocian, C. lenco, S. Chessa, y W. Grolman, «Intelligent smartphone audiometry», Advances in Intelligent Systems and Computing, vol. 615, pp. 112-120, 2017. 
[30] C. Louw, D. W. Swanepoel, R. H. Eikelboom, y H. C. Myburgh, «Smartphone-Based Hearing Screening at Primary Health Care Clinics», Ear Hear, vol. 38, n. ${ }^{\circ}$ 2, pp. e93-e100, abr. 2017.

[31] D. W. Swanepoel, «Enhancing ear and hearing health access for children with technology and connectivity», American Journal of Audiology, vol. 26, n. ${ }^{\circ}$ 3S, pp. 426-429, 2017.

[32] J. van Tonder, D. W. Swanepoel, F. Mahomed-Asmail, H. Myburgh, y R. H. Eikelboom, «Automated Smartphone Threshold Audiometry: Validity and Time Efficiency», J. Am. Acad. Audiol., vol. 28, n. ${ }^{\circ}$ 3, pp. 200-208, mar. 2017.

[33] S. Yousuf Hussein, D. W. Swanepoel, F. Mahomed, y L. Biagio de Jager, «Communitybased hearing screening for young children using an mHealth service-delivery model», Glob Health Action, vol. 11, n. ${ }^{\circ}$ 1, p. 1467077, 2018.

[34] J. L. Manganella, D. J. Stiles, K. Kawai, D. L. Barrett, L. W. O'Brien, y M. A. Kenna, «Validation of a portable hearing assessment tool: Agilis Health Mobile Audiogram», International Journal of Pediatric Otorhinolaryngology, abr. 2018.

[35] J.-M. Potgieter, D. W. Swanepoel, H. C. Myburgh, y C. Smits, «The South African English Smartphone Digits-in-Noise Hearing Test: Effect of Age, Hearing Loss, and Speaking Competence», Ear Hear, nov. 2017.

[36] T. Bright y D. Pallawela, «Validated Smartphone-Based Apps for Ear and Hearing Assessments: A Review.», JMIR rehabilitation and assistive technologies, vol. 3, n. ${ }^{o}$ 2, p. e13, dic. 2016.

[37] N. Nakamura, «Development of mobile audiometric test system using mobile phones», presentado en APBME 2003 - IEEE EMBS Asian-Pacific Conference on Biomedical Engineering 2003, 2003, pp. 356-357.

[38] S. Figueira, K. Nguyen, y S. Panditrao, «HearThat? - An app for diagnosing hearing loss», presentado en Proceedings of the 4th IEEE Global Humanitarian Technology Conference, GHTC 2014, 2014, pp. 518-524.

[39] N. Chauhan y J. Shah, «Smart Phone Based Audiometry in City Traffic Police», 2018. 